\title{
First detection of return currents in solar flares by spectropolarimetry with THEMIS
}

\author{
J.-C. Hénoux ${ }^{1}$ and M. Karlický² \\ ${ }^{1}$ Observatoire de Paris, LESIA/UMR8645, Place Jules Janssen, 92195 Meudon principal Cedex, France \\ e-mail: henoux@mesiob.obspm.fr \\ 2 Astronomical Institute, Academy of Sciences of the Czech Republic, 25165 Ondřejov, Czech Republic \\ e-mail: karlicky@asu.cas.cz
}

Received 10 December 2002 / Accepted 31 March 2003

\begin{abstract}
Using THEMIS French-Italien telescope with the MTR mode, the Hydrogen $\mathrm{H} \alpha$ and $\mathrm{H} \beta$ lines have been observed to be linearly polarized up to a few percent by impact during the impulsive phase of two solar flares associated with high-frequency radio pulses. Two privileged directions of linear polarization are present, respectively radial (in the disk center to flare direction) and tangential (perpendicular to the radial direction). This 90 degree change in the linear polarization direction is interpreted as due to the chromospheric return current generated by the penetration of a non-thermal electron beam into the chromosphere.
\end{abstract}

Key words. Sun: flares - plasmas - polarization

\section{Introduction}

In order to neutralize the local plasma during the bombardment of the solar chromosphere by particle beams, return currents are generated. In a preceding paper (Karlický \& Hénoux 2002), the impact $\mathrm{H} \alpha$ polarization due to return current electrons associated with beam electrons was computed. Here, we present examples of polarization measurements from two recent flares. These flares have been selected since they are associated with high-frequency radio pulses that indicate electron beam bombardment of the chromospheric layers. The polarization measurements show the presence of a return current induced by the electron beam.

\section{Impact line polarization}

\subsection{Main characteristics}

Atomic line excitation by collisions with electrons having anisotropic velocity distribution functions leads to linearly polarized line emission. The degree of linear polarization $P(\beta, E)$ of line radiation in a direction making an angle $\beta$ with the particles propagation direction (Percival \& Seaton 1959) is usually defined as:

$P(\beta, E)=\left(I_{\|}-I_{\perp}\right) /\left(I_{\|}+I_{\perp}\right)$,

where $I_{\|}$and $I_{\perp}$ are respectively the intensities of the vibrations parallel and perpendicular to the plane that contains the particle trajectory and the line of sight (see Fig. 1). The degree of

Send offprint requests to: J.-C. Hénoux,

e-mail: Jean-claude.henoux@obspm.fr

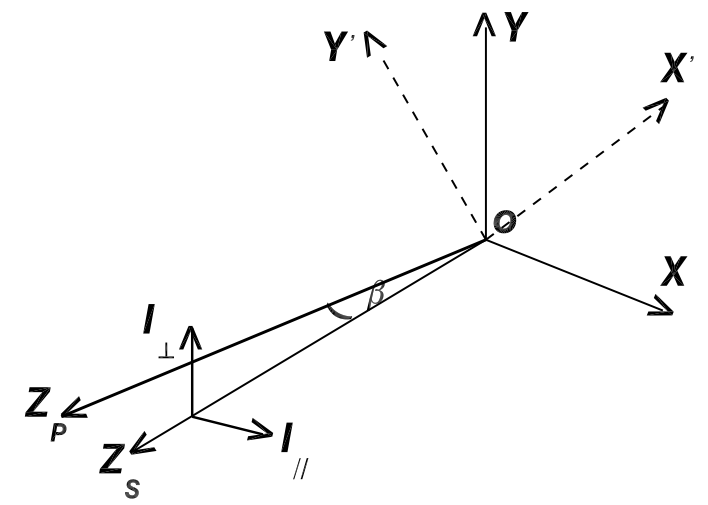

Fig. 1. Systems of coordinates related to the particle propagation direction $O Z_{P}$ and used in Sect. 2.1 to define the Stokes parameters.

polarization, $P\left(90^{\circ}, E\right)$, of the line radiation observed at $90^{\circ}$ to the beam depends on the transition and on the particle energy.

Atomic impact excitation in collisions with mono-energetic and mono-directional electrons of energy greater than $E_{\text {th }}-$ the threshold excitation energy - leads to polarized line radiation. At $E$ equal to $E_{\text {tov }}$ - the turn over energy - the sign of the degree of polarization changes. In the case of the $\mathrm{H} \alpha$ line, the degree of polarization changes from positive to negative values and $E_{\text {th }}$ and $E_{\text {tov }}$ are respectively equal to $12 \mathrm{eV}$ and $200 \mathrm{eV}$ (Hénoux \& Vogt 1998 and references therein).

Linear polarization of radiation can be characterized by the Stokes parameters, $I, Q$ and $U$. Selecting two $X Y Z_{S}$ and $X^{\prime} Y^{\prime} Z_{S}$ coordinate systems where $O Z_{S}$ is parallel to the line of sight direction and where the $X^{\prime} Y^{\prime} Z_{S}$ coordinate 

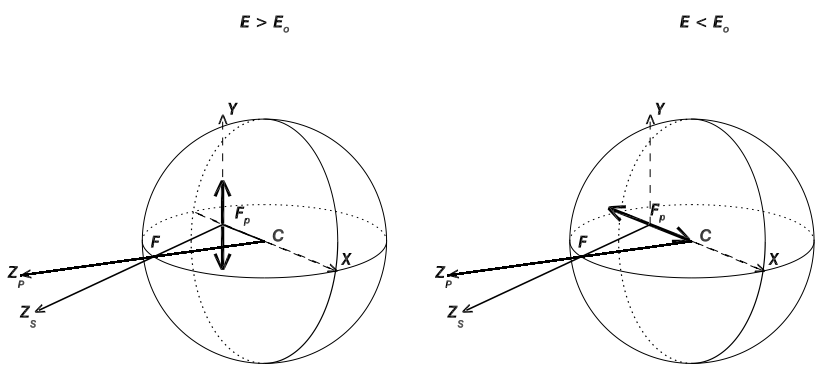

Fig. 2. Orientations of $\mathrm{H} \alpha$ line linear polarization for hydrogen excitation by collision with electrons moving along the solar vertical with energies lower or greater than $E_{0}=E_{\mathrm{tov}}$. For $E>(<) E_{\mathrm{tov}}, P$ is negative (positive) and the polarization direction is tangential (radial), i.e. perpendicular (parallel) to the flare to disk center direction.

system is derived from the $X Y Z_{S}$ coordinate system by a positive $45^{\circ}$ rotation around the $Z_{S}$ axis, $Q$ and $U$ are respectively defined as:

$$
\begin{aligned}
& Q(\beta, E)=I_{X}-I_{Y} \\
& U(\beta, E)=I_{X^{\prime}}-I_{Y^{\prime}} \\
& I(\beta, E)=I_{X}+I_{Y} .
\end{aligned}
$$

For a given direction of polarization, there is an infinity of possible settings for the positioning of the $O X Y$ (and associated $O X^{\prime} Y^{\prime}$ ) reference systems. By selecting, as shown in Fig. 1, a $X Y Z_{S}$ coordinate system such that $O X$ and $O Y$ are directions respectively parallel and perpendicular to the plane containing $O Z_{S}$ and the particle propagation direction $O Z_{P}$, the Stokes parameters $Q(\beta, E)$ and $U(\beta, E)$ in Eq. (2) of impactpolarized radiation can be rewritten for any line as:

$$
\begin{aligned}
& Q(\beta, E)=P(\beta, E) I(\beta, E) \\
& U(\beta, E)=0 .
\end{aligned}
$$

\subsection{Expected orientation of line impact linear polarization seen on the sun for mono-energetic electrons moving along the solar vertical direction}

As illustrated in Fig. 2 and as a consequence of Sect. 2.1, for a line with positive polarization at excitation threshold like $\mathrm{H} \alpha$ or $\mathrm{H} \beta$, depending on the particle energy $E$, the line linear polarization produced by particles moving along the solar vertical, either down like beam electrons or up, like associated return current electrons, is expected to be either tangential or radial. Whatever the position on the solar disk of particle impact, for $E$ greater than the turnover energy $E_{\text {tov }}$, the degree of polarization $P$ observed is negative and the polarization direction is tangential, i.e. perpendicular to the flare to the disk center direction. For $E$ lower than $E_{\mathrm{tov}}, P$ is positive and the polarization direction is radial, i.e. along the flare to disk center direction.

\section{Solar flare impact line polarization observations}

A few solar flares have been observed with THEMIS by using the MTR(MulTi-Raies) multi-lines spectropolarimetric mode in 2000 and 2001. We are reporting here the characteristics of

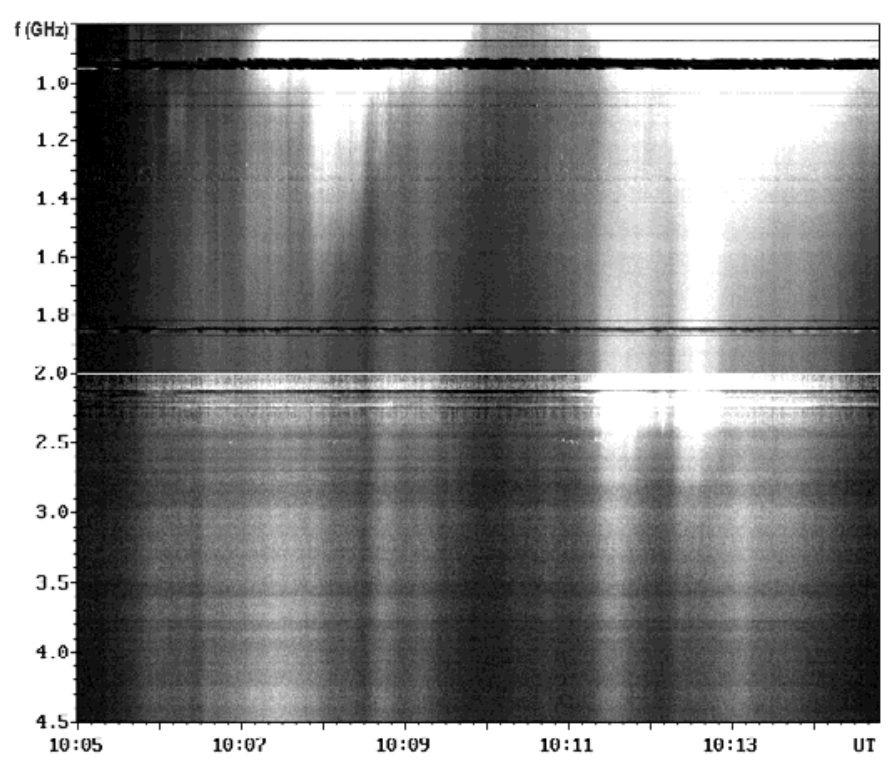

Fig. 3. The $0.8-4.5 \mathrm{GHz}$ radio spectrum observed during the June 15th 2001 flare by two Ondrejov radiospectrographs $(0.8-2.0 \mathrm{GHz}$ and $2.0-4.5 \mathrm{GHz}$ range).

the linear polarization observed in $\mathrm{H} \alpha$ and $\mathrm{H} \beta$ at some times and some locations during the impulsive phase of two solar flares. The main emphasis is put on the June 15th 2001 flare. Some characteristics of the impact polarization in July 18th 2000 flare are more briefly presented, since they show that observations made with a very different observational setting lead also to the same conclusions.

\subsection{June 15th 2001 flare}

The June 2001 observations used the MTR mode with one CCD camera per selected atomic line, on which both $I+S$ and $I-S$ spectra were focused. The Stokes parameter $S$ could be either $U$ or $Q$. It is defined in a $X Y Z_{S}$ coordinate systems fixed by the instrumental setting and given in the Appendix, together with the procedure used to reduce the data. The field of view along the spectrograph entrance slit direction is close to one arc minute.

A $1 \mathrm{~N}$ flare was observed on June 15, 2001. It was located at S26E41 in active region AR9502. The NOAA reported begin, maximum and end times are respectively 10:01, 10:13 and 10:20 UT. On radio waves (Fig. 3) the measured polarization was accompanied by a series of radio pulses in the $0.8-0.2 \mathrm{GHz}$ as well as in the $2.0-4.5 \mathrm{GHz}$ ranges. While the low-frequency part $(\leq 2 \mathrm{GHz})$ is probably generated by fast electron beams through plasma emission processes (their frequency drift is not measurable here due to a short density scale at this frequency range and the time resolution of $0.1 \mathrm{~s}$ only), the high-frequency part $(>2 \mathrm{GHz})$ is the gyro-synchrotron radiation of beam electrons having a velocity component perpendicular to the magnetic field. Simultaneously, the hard X-ray observation by the HXRS instrument (Fárník et al. 2001) indicates the presence of 24-100 keV electrons, the origin of the hard X-ray source (Fig. 4). These two types of observations can be considered as indications of electron beams bombarding the chromosphere. 


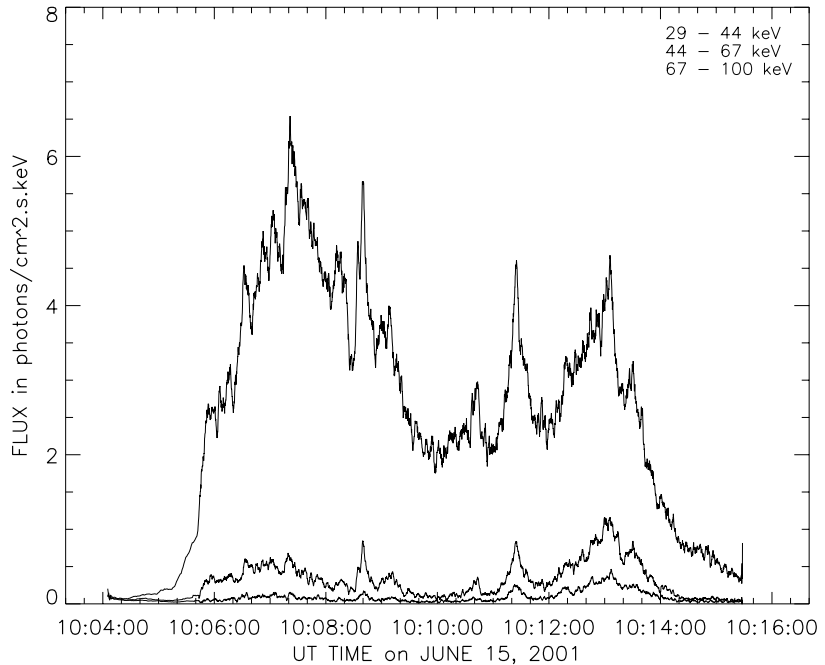

Fig. 4. Time variation of the Hard X-ray flux observed during the June 15th 2001 flare by HXRS, that also indicates electron beams. At any time, the highest flux corresponds to the lowest energy band.

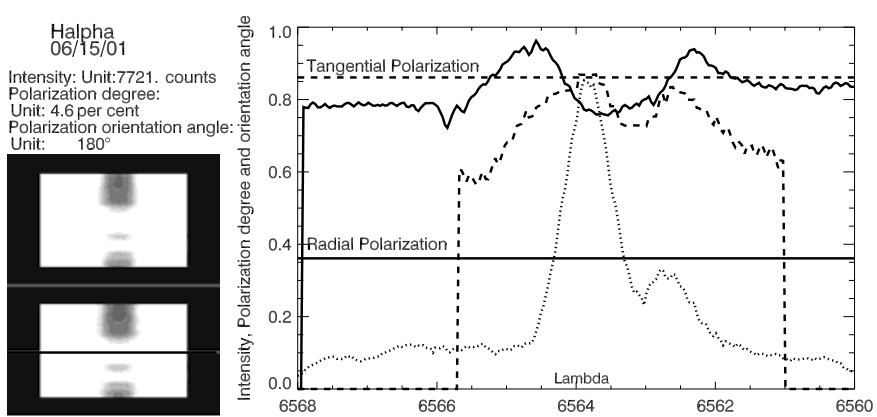

Fig. 5. $\mathrm{H} \alpha$ line intensity (full line), linear polarization degree (dotted line) and orientation profiles (dashed line) observed at 10:07:34 UT on June 15th 2001 at location $a$ (dark line on the lower left spectrum). The superposed two $I+S$ (up) and $I-S$ (down) spectral images in the spectrograph dispersion plane for one of the two Stokes parameters $U$ or $Q$ are shown on the left of the figure.

THEMIS observations started at 10:07:20 UT. Flare region scanning was made by translating the solar image perpendicularly to the entrance slit of the spectrograph. The scanning was made in twenty steps, separated by 3 arcsec, with a scanning time for these twenty steps close to $1 \mathrm{mn} 30 \mathrm{~s}$. A 1 arcsec entrance slit width was used. For each position, the Stokes parameters $Q$ and $U$ were successively recorded with a 2.3 s time interval. Five lines were observed simultaneously.

We report here on the $\mathrm{H} \alpha$ and $\mathrm{H} \beta$ line observations. Intensity, linear polarization degree and orientation profiles in these lines, at two flare locations called $a$ and $b$ made at two different times near the beginning of the impulsive phase, are presented. Regions $a$ and $b$ have been selected for the rather typical shapes of the $\mathrm{H} \alpha$ line profiles emitted, that show either a central absorption inside the emission core $(a)$ or a broad emission profile $(b)$. The profiles were obtained by integrating over about 2.5 arcsec along the slit direction. The positioning into the locations selected was the one corresponding to the brightest intensity profile and not to the highest polarization
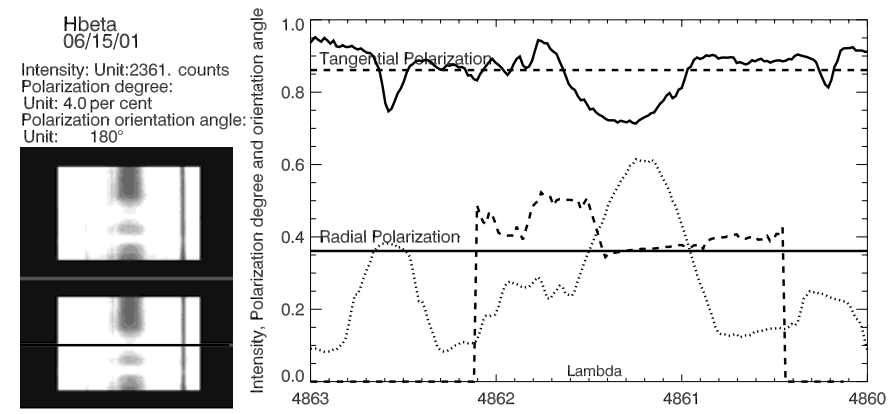

Fig. 6. $\mathrm{H} \beta$ line intensity (full line), linear polarization degree (dotted line) and orientation profiles (dashed line) observed at 10:07:34 UT on June 15th 2001 at location $a$.

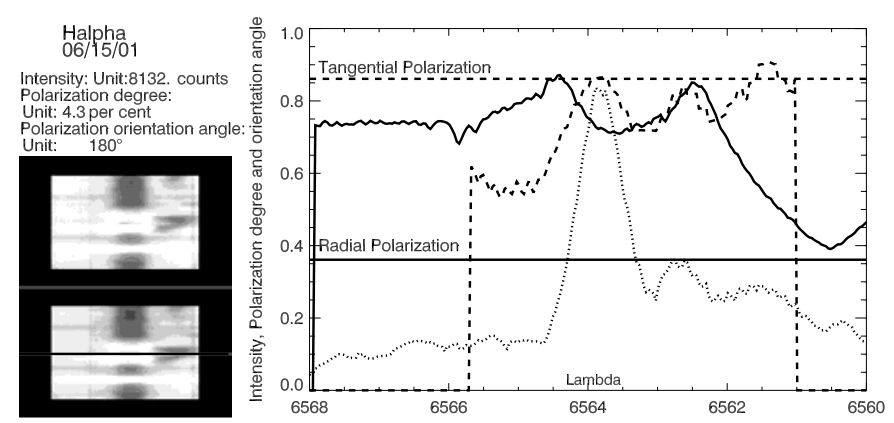

Fig. 7. $\mathrm{H} \alpha$ line intensity (full line), linear polarization degree (dotted line) and orientation profiles (dashed line) observed at 10:09:09 UT on June 15th 2001 at location $a$.

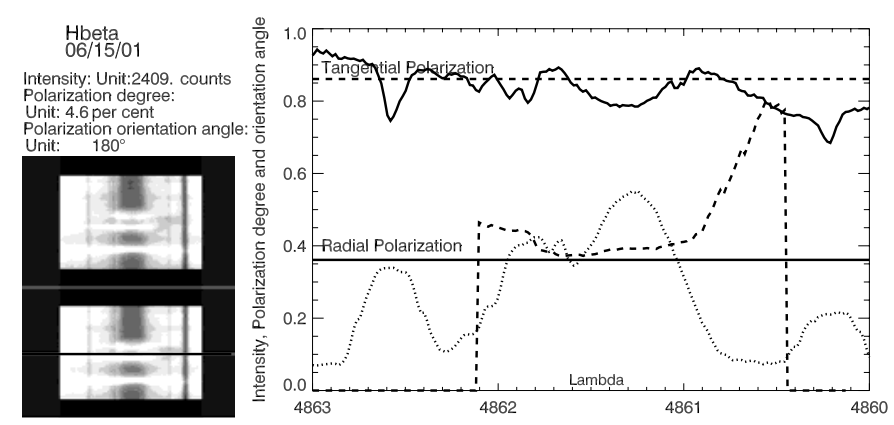

Fig. 8. $\mathrm{H} \beta$ line intensity (full line), linear polarization degree (dashed line) and orientation profiles (dashed line) observed at 10:09:09 UT on June 15th 2001 at location $a$.

signal. The profiles are plotted in Figs. 5-8 for location $a$ and in Figs. 9-12 for location $b$. The polarization orientation angle, shown in all figures quoted above, takes as its origin the celestial south-north direction. The disk center to flare direction made an angle with the reference axis close to $65^{\circ} \pm 5^{\circ}$ (close to 0.36 when $180^{\circ}$ is taken as unity, as in the figures quoted above). The wavelength interval over which the direction of polarization is plotted is limited in order not to include adjacent atomic lines and to be restricted to wavelengths where the polarization degree is higher than $0.5 \%$.

As shown in Fig. 5, at 10:07:34 UT, at location $a$, a rather narrow polarization peak (half width close to $0.10 \mathrm{~nm}$ ) reaching $3.9 \%$ is observed in the $\mathrm{H} \alpha$ line. The polarization direction is tangential. At the same time and at the same location, 

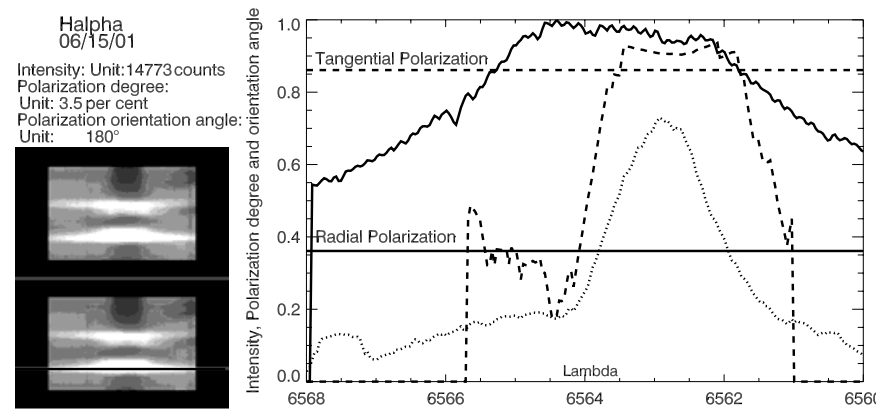

Fig. 9. $\mathrm{H} \alpha$ line intensity (full line), linear polarization degree (dotted line) and orientation profiles (dashed line) observed at 10:07:48 UT on June 15 th 2001 at location $b$.
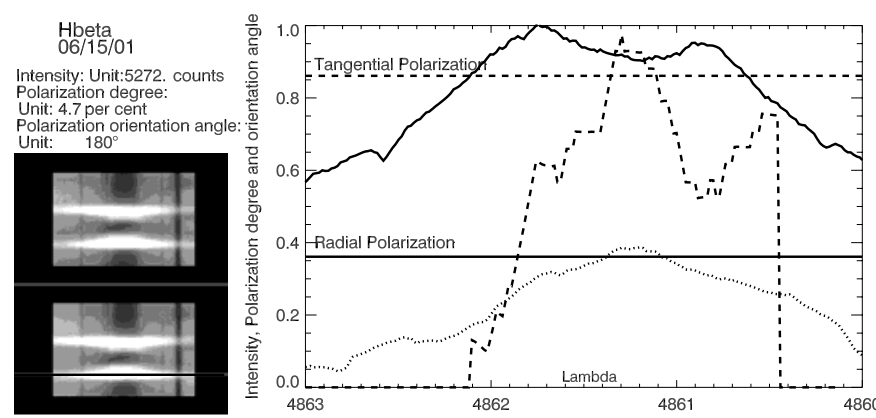

Fig. 10. $\mathrm{H} \beta$ line intensity (full line), linear polarization degree (dotted line) and orientation profiles (dashed line) observed at 10:07:48 UT on June 15 th 2001 at location $b$.

a $0.05 \mathrm{~nm}$ width polarization peak reaching $2.4 \%$ is present in $\mathrm{H} \beta$ (see Fig. 6). Contrary to $\mathrm{H} \alpha$, the $\mathrm{H} \beta$ polarization direction is radial. One and half minutes later, at 10:09:09 UT, higher intensities are present. The polarization intensity and orientation keep the same characteristics. The peaks in polarization degree reach respectively $3.6 \%$ and $2.4 \%$, at the same wavelength positions as previously, and the polarization directions are tangential and radial in $\mathrm{H} \alpha$ and $\mathrm{H} \beta$ respectively (Figs. 7 and 8). Since the anisotropy of the particle velocity distribution function decreases with depth, we expect the polarization to be higher at line center than in the wings as observed in Figs 5-8.

At location $b$, at 10:07:48 and 10:09:23 UT, the $\mathrm{H} \alpha$ and $\mathrm{H} \beta$ lines are very broad with line widths, constant during this time interval, of about $0.20 \mathrm{~nm}$ (see Figs. 9-12). The line intensities are about twice as intense as at location $a$. Polarization peaks are wider than at location $a$, reaching $0.2 \mathrm{~nm}$ in $\mathrm{H} \alpha$ and $0.15 \mathrm{~nm}$ in $\mathrm{H} \beta$ at 10:07:48 UT. The polarization degree peaks are lower than at location $a$. They reach only 2.4 and $1.4 \%$ in $\mathrm{H} \alpha$ and 1.8 and $0.8 \%$ over nearly $0.2 \mathrm{~nm}$ in $\mathrm{H} \beta$. This decrease of the polarization degree associated with an increase of the line intensity and width is a general feature present in all polarization observations. This is in agreement with an increase of the depolarization in a presumably denser chromospheric plasma at locations of higher line intensities. This depolarization results from the exchange of populations between the sub-levels that are induced by collisions with ambient electrons and neutral hydrogen atoms. The higher the local density, the higher the depolarization effect.

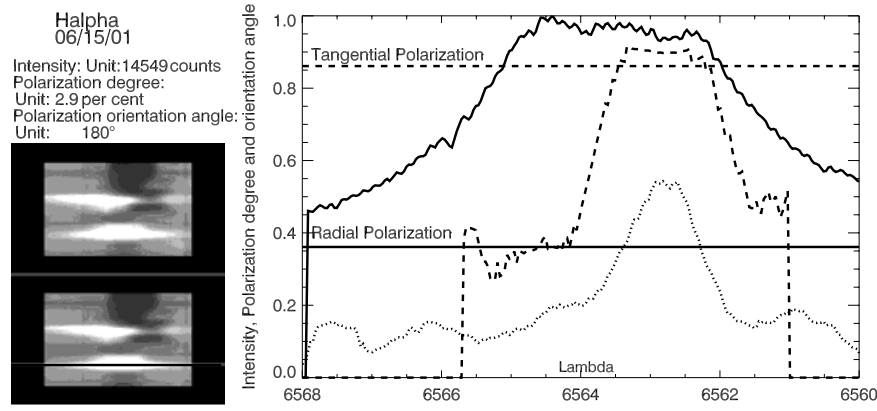

Fig. 11. H $\alpha$ line intensity (full line), linear polarization degree (dotted line) and orientation profiles (dashed line) observed at 10:09:23 UT on June 15th 2001 at location $b$.

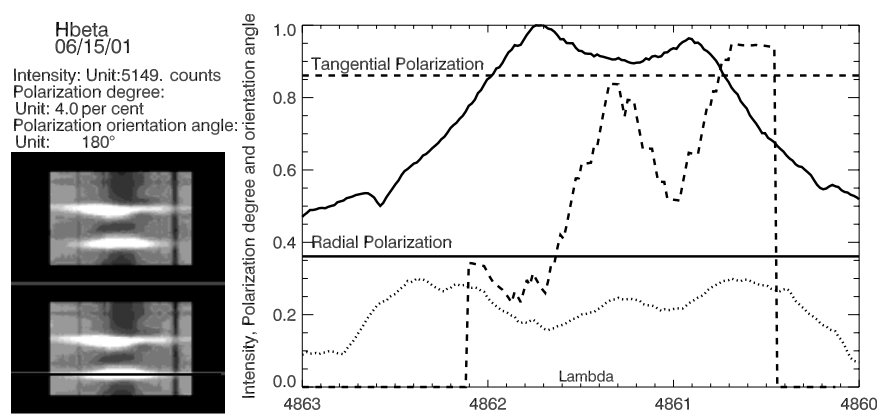

Fig. 12. $\mathrm{H} \beta$ line intensity (full line), linear polarization degree (dotted line) and orientation profiles (dashed line) observed at 10:09:23 UT on June 15th 2001 at location $b$.

At location $b$, the polarization direction is tangential in both lines. In $\mathrm{H} \alpha$, tangential polarization is present over $0.2 \mathrm{~nm}$ at 10:07:48 and $0.15 \mathrm{~nm}$ at 10:09:23 UT. The polarization direction signal is more noisy in $\mathrm{H} \beta$ being tangential over $0.2 \mathrm{~nm}$ only near polarization peaks (Fig. 8). As long as the relative velocities of the beam and return current particles compared to the excited hydrogen atoms stay in a domain where the polarization degree does not change sign, the line polarization direction is not affected by the local thermal or turbulent motions of these hydrogen atoms. We then expect the polarization direction to be constant over a wavelength interval larger than the polarization profile width. The wavelength positions of the polarization and intensity peaks are also expected to coincide, as nearly observed at location $a$, where a small shift to the red is present. However, at location $b$ the polarization peak is shifted towards the blue relative to the line and intensity peak. Moreover, in the $\mathrm{H} \alpha$ line at 10:07:48 UT and in both $\mathrm{H} \alpha$ and $\mathrm{H} \beta$ lines at 10:09:23 UT, the polarization direction changes from radial in the red wing to tangential in the blue wing. This may indicate that at location $b$ the relative velocity of the return current electrons compared to the excited hydrogen atoms is close to the threshold velocity as discussed in Sect. 5 .

\subsection{July 18 th 2000 flare}

July 2000 observations used the MTR mode with a grid. This grid is located just before the polarimeter and the solar image is formed nearly on the grid at the entrance of the polarimeter. The grid allows us to separate the images formed by the 


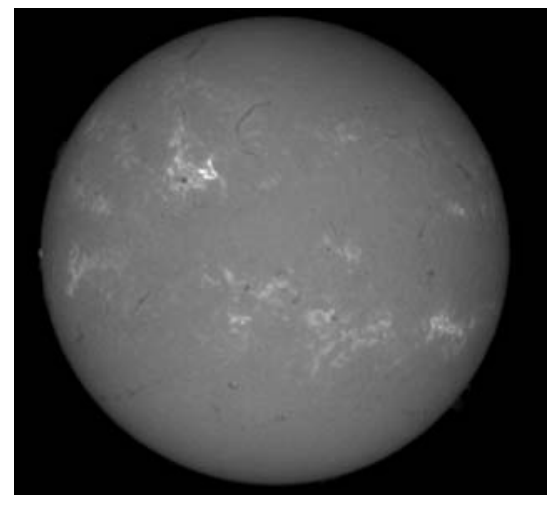

Fig. 13. Location of $\mathrm{H} \alpha$ flare observed at Paris Observatory (Meudon) at 7:22 UT on July 18 th 2000 .

extraordinary and ordinary beams that carry the $I+S$ and $I-S$ signals without additional optics. So the optical paths for the two beams are as identical as possible (Semel 1980). The opaque bands stop the solar light over a width of 16 arcsec on the sun. The grid transmission bands between them transmit the solar light through bands of 16 arcsec width of the solar image. The light beam so transmitted is split in two by the polarimeter and then focused on the entrance of the spectrograph. There, one of the two beams fills the gap produced by the opaque bands of the grid. Thus, the $I+S$ images of the transparent strips are located between the $I-S$ images of these strips. There are three sets of $I+S, I-S$ pairs.

A $1 \mathrm{~F}$ flare was observed on July 18th 2000 . This flare was located at S13E16 in active region AR9087 (see Fig. 13). NOAA reported the begin, maximum and end times to be respectively 07:08, 07:21 and 08:03 UT. As for the June 15th 2001 flare radio pulses in the $0.8-0.2 \mathrm{GHz}$ as well as in the 2.0-4.5 GHz ranges were observed by the Ondrejov radiospectrograph. THEMIS observations started at 07:22:02 UT just after the reported time of maximum emission. The scanning was made in twenty steps, separated by 4 arcsec, with a scanning time for these twenty steps close to $50 \mathrm{~s}$. A 1.2 arcec entrance slit width was used. The scans perpendicular to the slit direction were alternatively shifted by 16 arcsec (the angular width of a grid band) in the slit direction. For each observing position, $U$ and $Q$ were measured successively with a 2 s time interval.

Intensity and polarization profiles were obtained at various locations by integrating over 2 arcsec along the slit direction. Five lines were observed simultaneously. In order to show the agreement between June 15th 2001 and July 18th 2000 results, obtained with two different instrumental settings, we present in Figs. 14 and 15 a few examples of $\mathrm{H} \alpha$ and $\mathrm{H} \beta$ lines intensity, linear polarization degree and orientation profiles observed at five positions along the spectrograph slit near the maximum of the flare emission. As in the June 15th 2001 flare, the highest intensity peak does not correspond to the highest polarization peak. The observations were made at 7:22:52 UT on July 18 th 2000. The disk center to flare direction made an angle with the reference axis - the celestial south-north direction - close to $55^{\circ} \pm 5^{\circ}$. In $\mathrm{H} \alpha$, all along the bright location chosen, the linear polarization direction is very close to the tangential one, deviating only by a 10 degree clockwise rotation from this direction.
On the other hand the $\mathrm{H} \beta$ line linear polarization, tangential in one location, becomes radial in the other four locations. This change from tangential in $\mathrm{H} \alpha$ to radial in $\mathrm{H} \beta$ was also present in June 15th 2001 observations. As seen in Fig. 15, the tangential direction of polarization is associated with the lowest intensity and polarization degree at line center.

\section{Detailed analysis of atomic line linear polarization resulting from beam and associated return current impact excitation}

\subsection{Relative contributions of beam high energy electrons and return current electrons to line linear impact polarization}

The relative contribution of return current electrons to the line linear impact polarization depends on the return current electron velocity, which in turn depends on the model of return current used.

Neglecting all other line excitation processes, the Stokes parameters of any atomic line radiation resulting from excitation by collisions with both the electrons of energy $E_{\mathrm{B}}$ of a mono-energetic beam and the return current electrons of energy $E_{\mathrm{R}}$ are given, in a $X Y Z$ reference system defined as in Sect. 2, by:

$$
\begin{aligned}
& Q^{T}(\beta)=Q\left(\beta, E_{\mathrm{B}}\right)+Q\left(\beta, E_{\mathrm{R}}\right) \\
& U^{T}(\beta)=0 . \\
& I^{T}(\beta)=I\left(\beta, E_{\mathrm{B}}\right)+I\left(\beta, E_{\mathrm{R}}\right) .
\end{aligned}
$$

These equations can be rewritten as:

$Q^{T}(\beta)=P\left(\beta, E_{\mathrm{B}}\right) I\left(\beta, E_{\mathrm{B}}\right)+P\left(\beta, E_{\mathrm{R}}\right) I\left(\beta, E_{\mathrm{R}}\right)$

$U^{T}(\beta)=0$.

$I^{T}(\beta)=I\left(\beta, E_{\mathrm{B}}\right)+I\left(\beta, E_{\mathrm{R}}\right)$

By assuming that the intensity of the atomic line is proportional to its emissivity and by using the neutrality condition $n_{\mathrm{e}}^{\mathrm{B}} v_{\mathrm{B}}=$ $n_{\mathrm{e}}^{\mathrm{R}} v_{\mathrm{R}}$, Eqs. (5) can be rewritten as:

$Q^{T}(\beta)=\left(P\left(\beta, E_{\mathrm{B}}\right) \sigma\left(E_{\mathrm{B}}\right)+P\left(\beta, E_{\mathrm{R}}\right) \sigma\left(E_{\mathrm{R}}\right)\right) n_{\mathrm{e}}^{\mathrm{B}} v_{\mathrm{B}}$

$I^{T}(\beta)=\left(\sigma\left(E_{\mathrm{B}}\right)+\sigma\left(E_{\mathrm{R}}\right)\right) n_{\mathrm{e}}^{\mathrm{B}} v_{\mathrm{B}}$,

where $\sigma(E)$ is the cross section for excitation in collisions with electrons of energy $E$ of the upper level of the atomic line transition considered.

For $E_{\mathrm{R}}<E_{\text {th }}$, only beam electrons contribute to the generation of impact polarization. In that case $Q^{T}(\beta)$ is negative, i.e. the polarization direction is tangential. Then for $E_{\mathrm{R}} \geq E_{\mathrm{th}}$, as shown below, the direction of linear polarization depends mainly on the energy of the return current electrons.

Under the Born approximation, at high energies $\sigma(E)$ is approximately inversely proportional to the electron energy. Therefore $\sigma\left(E_{\mathrm{B}}\right)=\sigma\left(E_{\mathrm{R}}\right) E_{\mathrm{R}} / E_{\mathrm{B}}$, where $E_{\mathrm{B}}$ is the local beam electron energy and Eqs. (6) can be rewritten as

$$
\begin{aligned}
& Q^{T}(\beta) \propto\left[P\left(\beta, E_{\mathrm{R}}\right)+P\left(\beta, E_{\mathrm{B}}\right) \frac{E_{\mathrm{R}}}{E_{\mathrm{B}}}\right] \sigma\left(E_{\mathrm{R}}\right) n_{\mathrm{e}}^{\mathrm{B}} v_{\mathrm{B}} \\
& I^{T}(\beta) \propto\left[1+\frac{E_{\mathrm{R}}}{E_{\mathrm{B}}}\right] \sigma\left(E_{\mathrm{R}}\right) n_{\mathrm{e}}^{\mathrm{B}} v_{\mathrm{B}}
\end{aligned}
$$



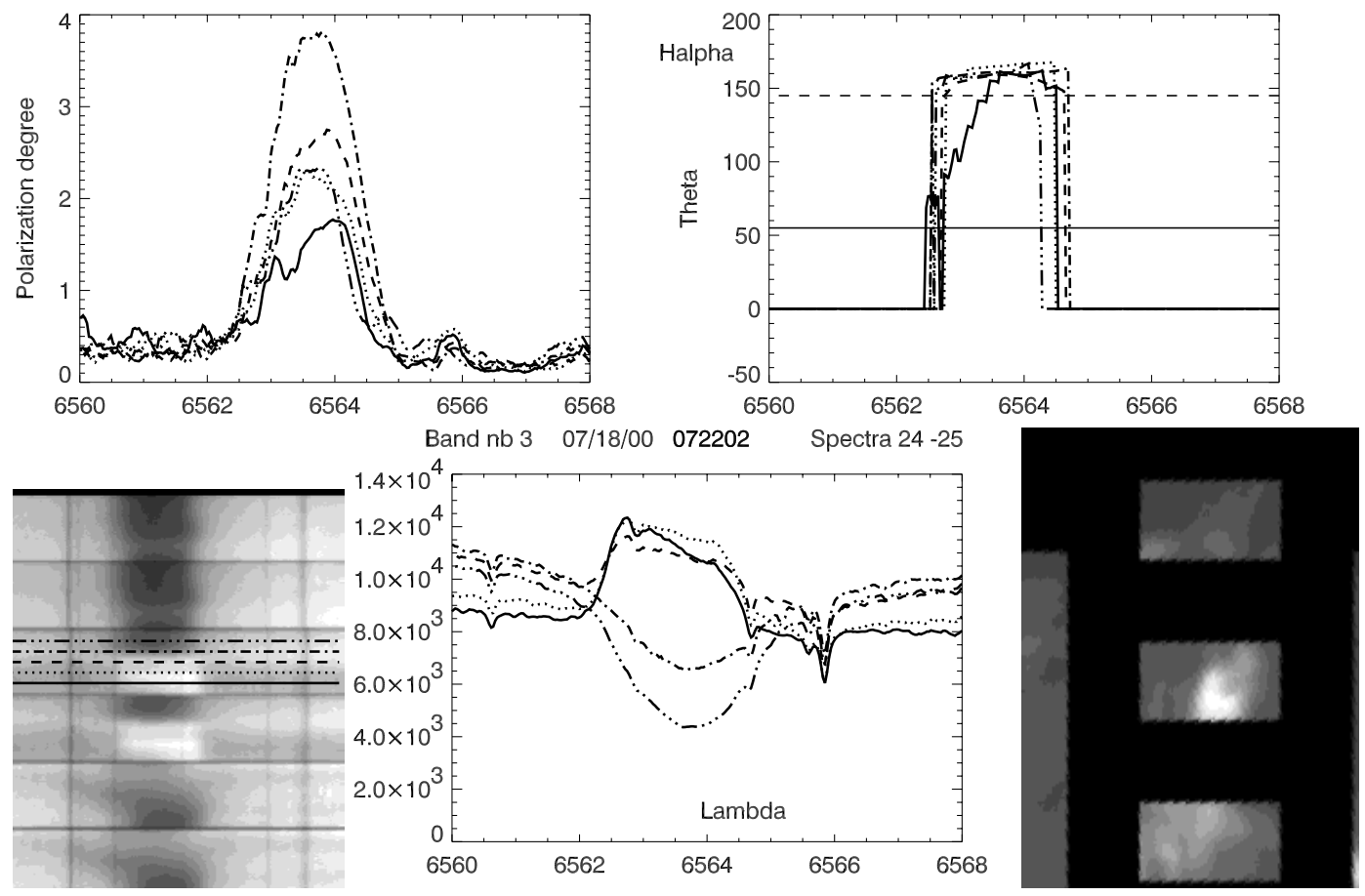

Fig. 14. $\mathrm{H} \alpha$ line intensity, linear polarization degree and orientation profiles at five positions (seen on bottom left part of the figure) along the spectrograph slit. Superposed on the left are three pairs of associated $I+S$ (up) and $I-S$ (down) spectral images in the spectrograph dispersion plane for a transmission band of the grid. The Stokes parameter used in this figure could be either $U$ or $Q$. Observation made at 7:22:52 UT on July 18 th 2000 .
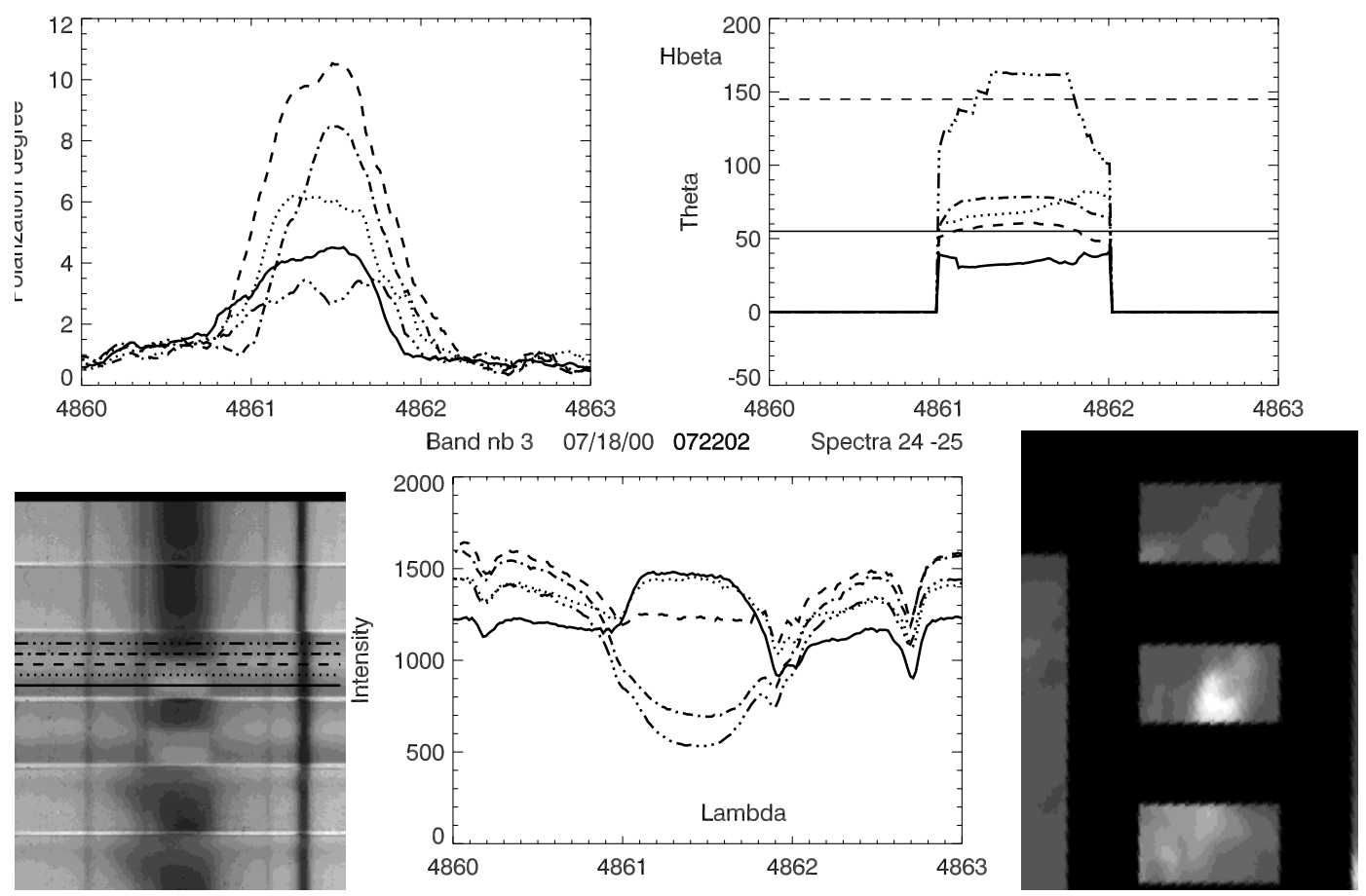

Fig. 15. $\mathrm{H} \beta$ line intensity, linear polarization degree and orientation profiles at five positions (seen on bottom left part of the figure) along the spectrograph slit. Observation made at 7:22:52 UT on July 18th 2000.

leading to a polarization degree:

$P^{T}(\beta)=\frac{Q^{T}(\beta)}{I^{T}(\beta)}=\frac{P\left(\beta, E_{\mathrm{R}}\right)+P\left(\beta, E_{\mathrm{B}}\right) \frac{E_{\mathrm{R}}}{E_{\mathrm{B}}}}{1+\frac{E_{\mathrm{R}}}{E_{\mathrm{B}}}}$.
In all plausible return current models $\frac{E_{\mathrm{R}}}{E_{\mathrm{B}}} \ll 1$, therefore

$P^{T}(\beta)=P\left(\beta, E_{\mathrm{R}}\right)$.

Consequently, for $E_{\mathrm{R}}>E_{\mathrm{tov}}$ or $E_{\mathrm{R}}<E_{\mathrm{th}}, Q^{T}(\beta)$ is negative (tangential direction of polarization). For $E_{\mathrm{th}}<E_{\mathrm{R}}<E_{\mathrm{tov}}$, 
$Q^{T}(\beta)$ is positive (radial direction of polarization). However, near the excitation threshold, where the Born approximation does not apply, the weight of the contribution of beam electrons to the polarization degree will be higher than the $\frac{E_{\mathrm{R}}}{E_{\mathrm{B}}}$ ratio.

\subsection{Return current electron energy and non-thermal electron energy flux}

We assumed that the return current is co-spatial with the electron beam. The relationship between the electron beam characteristics and the $\mathrm{H} \alpha$ line impact linear polarization direction is dependant on the return current model used. In simplified models (Hoyng et al. 1976; van den Oord 1990) the return current was considered as being formed by all background plasma electrons moving with the same drift speed. However, a detailed analysis of Rowland \& Vlahos (1983) revealed that the electrons carrying the return current could be limited to the electrons of high velocity in the local thermal plasma. Therefore, in the following we start first with the simple model where the return current is formed by all background plasma electrons. Then the model where the return current is carried by runaway electrons only is presented. Finally the effects of plasma waves and backscattered beam electrons on these processes are mentioned.

\subsubsection{Return current carried by all background electrons}

For a mono-energetic beam, it follows from the continuity equation that the beam particle number flux $\Phi_{\mathrm{p}}=n_{\mathrm{e}}^{\mathrm{B}} v_{\mathrm{B}}$ is conserved along its trajectory in the solar atmosphere. Here, it is assumed that the return current is carried by all background plasma electrons. Consequently, the velocity of the return current electrons can be expressed as:

$v_{\mathrm{R}}=\frac{\Phi_{\mathrm{p}}}{n_{\mathrm{e}}}=\frac{\Phi_{E}}{n_{\mathrm{e}} E_{0}}$,

where $n_{\mathrm{e}}$ is the density of background plasma, $\Phi_{E}$ is the initial energy beam flux and $E_{0}$ is the initial energy of beam electrons. Then the energy of the return current electrons is

$E_{\mathrm{R}}(\mathrm{eV})=110 .\left(\frac{\Phi_{E}\left(\mathrm{erg} \mathrm{cm}^{-2} \mathrm{~s}^{-1}\right)}{n_{\mathrm{e}}\left(\mathrm{cm}^{-3}\right) E_{0}(\mathrm{keV})}\right)^{2}$.

The relationship between the return current electrons energy and the local plasma density in the solar atmosphere, for three energy beam fluxes $\left(\Phi_{E}=10^{10}, 10^{11}\right.$, and $\left.10^{12} \mathrm{erg} \mathrm{cm}^{-2} \mathrm{~s}^{-1}\right)$ and two initial beam electron energies $\left(E_{0}=10\right.$ and $\left.20 \mathrm{keV}\right)$, is shown in Fig. 16. The ranges of radial and tangential polarizations are designated by " $\mathrm{R}$ " and "T". The low energy boundary of the radial polarization region is given by the hydrogen atomic level 3 excitation energy. Below this energy the polarization is only due to beam electrons. On the other hand, the high boundary of the radial polarization corresponds to the turn over energy. From Eq. (10), it can be seen that the return current electrons energy depends not only on the energy beam flux $\Phi_{E}$, but also on the initial beam electron energy $E_{0}$ and on the local electron number density $n_{\mathrm{e}}$. The $\Phi_{E} / n_{\mathrm{e}}$ dependance is plotted in Fig. 17 for two values of $E_{0}$. A monotonic increase of the return electron energy with $\Phi_{E} / n_{\mathrm{e}}$ increase is shown. There

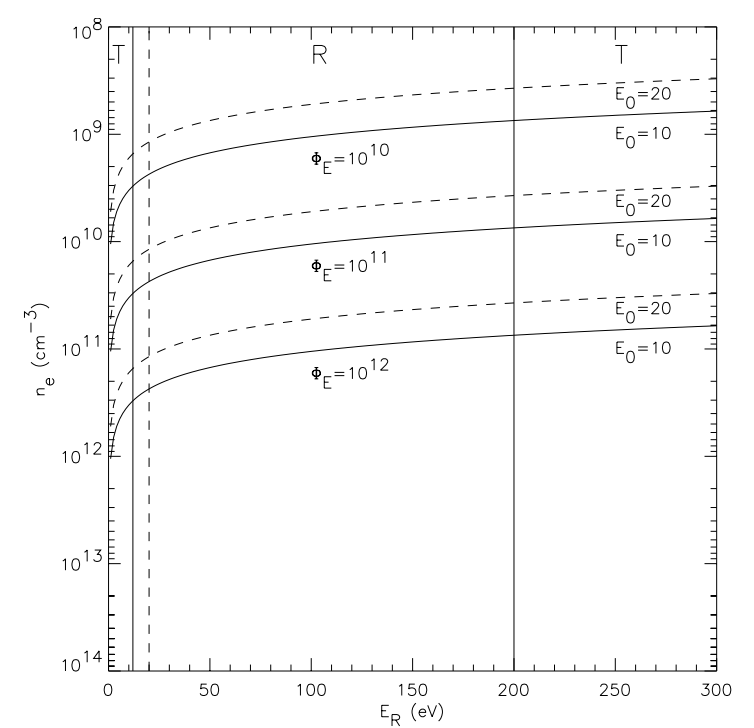

Fig. 16. Return current electron energy $E_{\mathrm{R}}$ as a function of the local electron number density $n_{\mathrm{e}}$ for various values of the beam initial energy flux $\Phi_{E}$ and electron beam initial energy $E_{0}$ in the model where the return current is formed by all background plasma electrons. Full line: $E_{0}=10 \mathrm{keV}$, dashed line: $E_{0}=20 \mathrm{keV}$. T and R are regions of respectively tangential and radial polarization. These regions are separated by full vertical lines. The dashed vertical line designates the energy of return current electrons corresponding to maximum polarization.

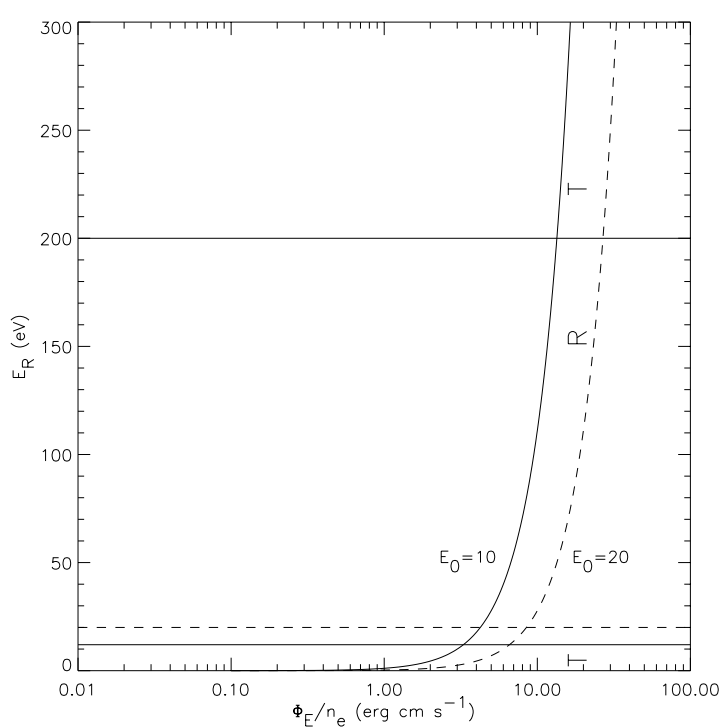

Fig. 17. Return current electron energy $E_{\mathrm{R}}$ as a function of the ratio of the beam initial energy flux $\Phi_{E}$ to the local electron number density $n_{\mathrm{e}}$ for two values of the electron beam initial energy $E_{0}$ in the model where the return current is formed by all background plasma electrons. Full line: $E_{0}=10 \mathrm{keV}$, dashed line: $E_{0}=20 \mathrm{keV}$. T and $\mathrm{R}$ are regions of respectively tangential and radial polarization; these regions are separated by full horizontal lines. The dashed horizonal line designates the energy of return current electrons corresponding to maximum polarization.

are two values of $\Phi_{E} / n_{\mathrm{e}}$ at which the polarization orientation changes either from tangential $(\mathrm{T})$ to radial $(\mathrm{R})$ polarization or from radial to tangential when $\Phi_{E} / n_{\mathrm{e}}$ decreases. 


\subsubsection{Return current carried by runaway electrons}

Now, let us assume that the return current is carried by the runaway electrons only. In this case, the electric currents neutralization condition is expressed as:

$v_{\mathrm{R}} N_{\text {run }}=n_{\mathrm{e}}^{\mathrm{B}} v_{\mathrm{B}}$,

where $N_{\text {run }}$ is the number density of run-away electrons, which can be estimated as (Norman \& Smith 1978):

$N_{\text {run }} \approx \frac{1}{2} n_{\mathrm{e}} \exp \left(-\frac{1}{2} \frac{\mathcal{E}_{\mathrm{Dr}}}{\mathcal{E}}\right)$

where

$\mathcal{E}_{\mathrm{Dr}}=\frac{m_{\mathrm{e}} v_{\mathrm{Te}}}{\tau_{\mathrm{ei}} e}$

is the Dreicer electric field. $m_{\mathrm{e}}$ and $e$ are the electron mass and charge. $v_{\mathrm{Te}}$ is the thermal velocity of the background plasma, and $\tau_{\mathrm{ei}}$ is the electron-ion collision time. The number of runaway electrons strongly depends on the ratio of this Dreicer electric field to the electric field $\mathcal{E}$ associated with the return current and beam current densities $j_{\mathrm{R}}$ and $j_{\mathrm{b}}$ :

$\mathcal{E}=\eta j_{\mathrm{R}}=\eta j_{\mathrm{b}}=\frac{m_{\mathrm{e}}}{n_{\mathrm{e}} e^{2} \tau_{\mathrm{ei}}} e v_{\mathrm{B}} n_{\mathrm{e}}^{\mathrm{B}}$,

where $\eta$ is the classical resistivity due to collisions.

Using the formulas mentioned above, the return-current electrons energy can be written as

$E_{\mathrm{R}}(\mathrm{eV})=2.84 \times 10^{-16}\left(\frac{\Phi_{E}}{\frac{1}{2} n_{\mathrm{e}} E_{0} \exp \left(-\frac{v_{\mathrm{Te}} n_{\mathrm{e}} E_{0}}{2 \Phi_{E}}\right)}\right)^{2}$,

where $\Phi_{E}$ and $E_{0}$ are respectively in erg $\mathrm{cm}^{-2} \mathrm{~s}^{-1}$ and erg. This relation was used to draw similar plots (Figs. 18 and 19) as for the previous model (Figs. 16 and 17), but only in the range where the induced electric field $\mathcal{E}$ is lower than half the Dreicer electric field $\mathcal{E}_{\mathrm{Dr}}$. Namely, for $\mathcal{E}$ comparable to $\mathcal{E}_{\mathrm{Dr}}$ the number of electrons forming the return current becomes comparable to the total number of electrons and the return current model becomes similar to the previous one with all background plasma electrons involved in the return current. For the limiting value of the electric field $\mathcal{E}$ taken equal to $\mathcal{E}_{\mathrm{Dr}} / 2$, the return current electrons carry a minimum of energy, $E_{\min }$, such that:

$E_{\min }(\mathrm{eV})=3.17 \times 10^{-4} \mathrm{~T}$,

we used for $T$, temperature of the background chromospheric plasma, the value $T=10^{4} \mathrm{~K}$.

The two models used lead to quite different return current energy electron number dependencies that, as discussed below, mutually agree with each other. For low beam energy fluxes, a small electric field is generated and a small number of high velocity run-away electrons is enough to neutralize the beam current. When the beam flux increases, the number of runaway electrons required for neutralization increases and electrons with lower energy are involved in the return current. The implication of minimum energy return current electrons takes place for $E=E_{\mathrm{Dr}} / 2$. At this electric field value, a bulk part of

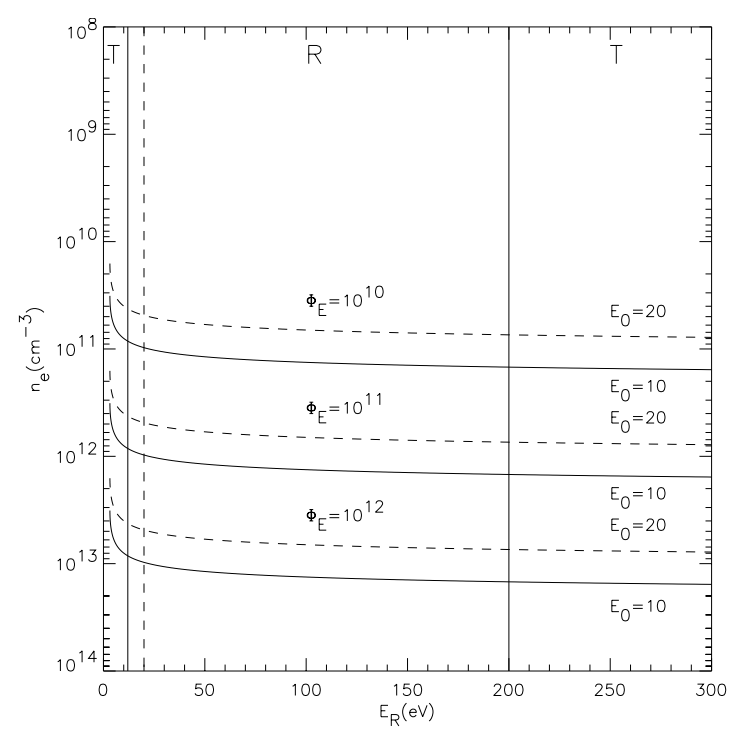

Fig. 18. Return current electron energy $E_{\mathrm{R}}$ as a function of the local electron number density $n_{\mathrm{e}}$ for various values of the beam energy flux $\Phi_{E}$ and electron beam energy $E_{0}$ in the model with the return current formed by run-away electrons. The notations are the same as in Fig. 16.

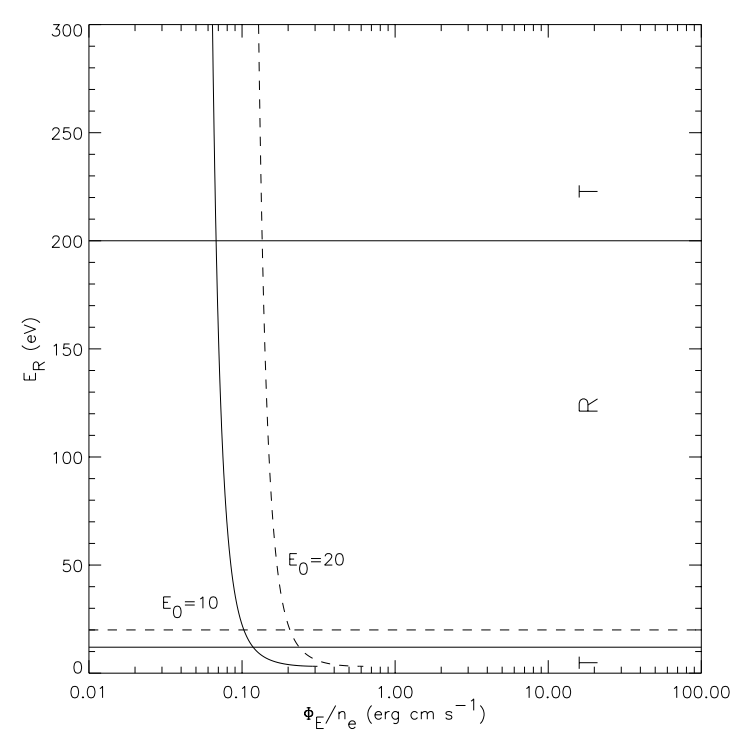

Fig. 19. Return current electron energy $E_{\mathrm{R}}$ as a function of of a ratio of the energy beam flux $\Phi_{E}$ and the local electron number density $n_{\mathrm{e}}$ for two values of the electron beam energy $E_{0}$ in the model with the return current formed by run-away electrons. The notations are the same as in Fig. 17.

electrons in the Maxwell distribution function starts to be accelerated and thus this regime is close to the regime where all electrons are involved in the return current (first model). The two values of $\Phi_{E} / n_{\mathrm{e}}$ at which polarization changes of direction that are obtained with the "run-away" model, correspond to much smaller beam energy fluxes than the ones given by the model that involves all background plasma electrons. 


\subsubsection{Effects of plasma waves and backscattered electrons}

In both models mentioned, some fraction of the background plasma electrons or all of them have an energy $(12-300 \mathrm{eV})$ such that their speed $\left(2 \times 10^{8}-1 \times 10^{9} \mathrm{~cm} \mathrm{~s}^{-1}\right)$ is much greater than the electron thermal velocity of the chromosphere (at $10000 \mathrm{~K}, v_{\mathrm{Te}} \sim 3.89 \times 10^{7} \mathrm{~cm} \mathrm{~s}^{-1}$ ). Therefore, the generation of plasma waves (e.g. ion-sound waves) is highly probable (see e.g. Heyvaerts 1981). In accordance with the paper by Rowland \& Vlahos (1983), we can expect that the bulk electrons of the background plasma are trapped in these waves. Then, only "run-away" electrons can carry the return current. This case is very similar to the collisional run-away electron case analyzed in the previous paragraph.

There is an another effect that could influence these processes. Namely, in the chromosphere some beam electrons can be backscattered in collisions and then could contribute to the return current (Karlický 1993).

\section{Discussion and conclusion}

The tangential direction of polarization observed in $\mathrm{H} \alpha$ could be generated either by deca $\mathrm{keV}$ electrons or by protons. However, this last hypothesis would require protons to reach the chromosphere with an energy slightly above $400 \mathrm{keV}$. That requires their initial energy to be greater than $1.25 \mathrm{MeV}$ (assuming a coronal mass of $1.36 \times 10^{-4} \mathrm{~g} \mathrm{~cm}^{-2}$ as in the Machado et al. 1980 flare model F1). Any power-law proton energy distribution with a low energy cut-off lower than $1.25 \mathrm{MeV}$ would not generate tangential polarization. The low energy protons, that dominate the proton number flux, would reach the chromosphere with an energy below $400 \mathrm{keV}$ and generate radial line polarization. On the other hand, if beam electrons cannot by themselves produce radial polarization, being strongly scattered when their energy becomes lower than $200 \mathrm{eV}$, they can generate tangential polarization. The presence of impulsive hard X-ray emission and/or radio pulses in the events reported here, together with a tangential orientation of the $\mathrm{H} \alpha$ line, supports atmospheric bombardment by deca $\mathrm{keV}$ electrons.

$\mathrm{H} \beta$ and $\mathrm{H} \alpha$ line polarization turnover energies $E_{\text {tov }}$ are the same (Feautrier 2002). Since $\mathrm{H} \beta$ is formed at lower heights in the solar atmosphere than $\mathrm{H} \alpha$, the change of the polarization direction from tangential in $\mathrm{H} \alpha$ to radial in $\mathrm{H} \beta$ is presumably due to the difference in depths of their formation layers. At a flare chromospheric electron number density equal to $10^{11} \mathrm{~cm}^{-3}$ the deceleration distance for $400 \mathrm{keV}$ protons is of about $200 \mathrm{~km}$, being close to the thickness of the $\mathrm{H} \beta$ line formation layer. The beam electrons deceleration distance is much shorter, being of the order of $100 \mathrm{~m}$ for $200 \mathrm{eV}$ electrons, and the observed change in polarization direction from tangential in $\mathrm{H} \alpha$ to radial in $\mathrm{H} \beta$ cannot be explained by the beam electron deceleration. However, as discussed below, the return current in association with the electron beam can explain this change.

Due to the electron beam energy losses in collisions and to the electron number density increase with depth, we expect the ratio $\Phi_{E} / n_{\mathrm{e}}$ to decrease with depth. Thus, the local value of $\Phi_{E} / n_{\mathrm{e}}$ must be lower in the $\mathrm{H} \beta$ line core forming layers than in the $\mathrm{H} \alpha$ line core forming layers. Depending on the assumed value of the initial electron energy (10 or $20 \mathrm{keV})$, Figs. 17 and 19 show two values of $\Phi_{E} / n_{\mathrm{e}}$ at which the transition from " $T$ " to "R" polarization occurs when $\Phi_{E} / n_{\mathrm{e}}$ decreases. They are respectively: $\Phi_{E} / n_{\mathrm{e}}=13.4$ at $E_{0}=10 \mathrm{keV}$ and $\Phi_{E} / n_{\mathrm{e}}=26.8$ at $E_{0}=20 \mathrm{keV}$ in the model with all background electrons, and $\Phi_{E} / n_{\mathrm{e}}=0.115$ at $E_{0}=10 \mathrm{keV}$ and $\Phi_{E} / n_{\mathrm{e}}=0.233$ at $E_{0}=20 \mathrm{keV}$ in the model with run-away electrons. Since it requires a lower energy flux, the second case is more probable. However, reality is presumably somewhere between these two cases since both models can be considered only as limiting cases.

The $\Phi_{E} / n_{\mathrm{e}}$ value can be estimated independently using hard X-ray observations and compared to the values derived from polarization measurements. Using the hard X-ray flux measured by HXRS and assuming thick target emission and an energy dependence given by a power law for electrons with a $20 \mathrm{keV}$ low energy cut-off, a total energy flux at the time of the reported observations of the order of $2.8 \times 10^{29} \mathrm{erg} \mathrm{s}^{-1}$ is derived. The area $A$ covered by the chromospheric flare is $10^{18} \mathrm{~cm}^{2} \leq A \leq 10^{19} \mathrm{~cm}^{2}$. By taking a mean estimate of $3 \times 10^{11} \mathrm{~cm}^{-3}$ as an intermediate value for $n_{\mathrm{e}}$ at the interface between the $\mathrm{H} \alpha$ and $\mathrm{H} \beta$ core forming atmospheric layers, the $\Phi_{E} / n_{\mathrm{e}}$ ratio is found to be $0.1 \leq \Phi_{E} / n_{\mathrm{e}} \leq 1$. The lower part of this range of values, which is also the most realistic, fits well with the runaway return current model that requires $\Phi_{E} / n_{\mathrm{e}}=0.233$ at $E_{0}=20 \mathrm{keV}$ to explain polarization observations.

Other observational facts support the hypothesis that the tangential polarization in the events reported here is due to beam electrons directly. We noticed in Sect. 3.2 that the tangential direction of polarization is associated with the lowest intensity and polarization degree at line center (Fig. 15), in agreement with the line excitation being due only to beam electrons that are less efficient than return current electrons (see Sect. 4.1). Moreover in Sect. 3.1, we noticed that in $\mathrm{H} \alpha$ at location $b$ in the June 15th 2001 flare at 10:07:48 UT and in both $\mathrm{H} \alpha$ and $\mathrm{H} \beta$ lines at 10:09:23 UT, the polarization direction is not constant along the line profile, being tangential in the blue and radial in the red parts of the line. This could happen when the energy of the chromospheric thermal plus turbulent motions is comparable to the line excitation threshold energy $E_{\mathrm{th}}$. Then, the relative velocity difference between return current electrons and hydrogen atoms may play a role for return current electrons of energy below but close to $E_{\text {th }}$. A positive relative velocity of $10^{7} \mathrm{~cm} \mathrm{~s}^{-1}$ (about $0.20 \mathrm{~nm}$ Doppler shift) of the hydrogen atoms relative to return current electrons of velocity $2 \times 10^{8} \mathrm{~cm} \mathrm{~s}^{-1}$ does represent an increase (relative to the case of hydrogen atoms at rest) in the energy available in the collision $\Delta E / E=2 \Delta v / v$ equal to $10^{-1}$, high enough to exceed the threshold energy $E_{\mathrm{th}}$. Consequently in the red part of the central line intensity profile, return current generated polarization competes with the beam generated one, leading to the faint radial polarization signal observed there (Figs. 9 and 11) in opposition to the tangential polarization present in the blue part of the profile. 
Thus, the impact polarization observations reported here provide support to the hypothesis that return currents are present in association with electron beams in impulsive flares.

Acknowledgements. M.K. would like to acknowledge the support of the Grants A3003202 and S1003006 of the Academy of Sciences of the Czech Republic. The other author wishes to thank THEMIS staff for assistance during observations in 2000 and 2001. Both authors wish to thank the French National Center for Scientific Research (CNRS) and the Academy of Sciences of the Czech Republic. Furthermore, both authors thank both staffs of the Solar Departments at Paris and Ondrejov Observatories for their support and kind hospitality. They also wish to thank the anonymous referee for comments and suggestions.

\section{Appendix A: Data reduction procedure}

All flare and flat-field observations have been corrected for the dark current contributions. Then, the signal differences resulting from differences in detector response $R_{C C D}$ and optics transmission $T_{\text {opt }}$ were eliminated by using "flat-fields". These flat fields were obtained by summing about 200 spectra obtained near disk center. The summation was made in order to wash out any solar brightness structure in the images. The ratio $R_{C C D}(i, j) T_{\mathrm{opt}}(i, j) / R_{C C D}\left(i^{\prime}, j^{\prime}\right) T_{\mathrm{opt}}\left(i^{\prime}, j^{\prime}\right)$ was computed for all pairs of pixels $(i, j)$ and $\left(i^{\prime}, j^{\prime}\right)$, where $i(\lambda)$ and $j(y)$ are pixel numbers, along respectively the dispersion and the slit directions, for the same wavelength $\lambda$ and location $y$ on the sun.

First, the $\left(j, j^{\prime}\right)$ association was derived using continuum intensity measurements not significantly affected by line curvature. For both $I-S$ and $I+S$ images, the intensity in the very far wings of the $\mathrm{H} \alpha$ line integrated over $0.20 \mathrm{~nm}\left(I_{\Delta \lambda}(y)\right)$ was computed. Then, $I_{\Delta \lambda}(y)$ was plotted as a function of distance $y$ (in pixels) along the spectrograph entrance slit for image $I-S$ together with $I_{\Delta \lambda}(y-(\Delta y+\mathrm{d} y))$ for the image $I+S$. As shown in Fig. A.1, for the June 15th 2001 flare the best correlation is found for $\Delta y+\mathrm{d} y$ equal to 142.2 pixels. The relative positioning accuracy in $y$ is 0.2 pixel $(0.08 \operatorname{arcsec})$.

In order to derive the $\left(i, i^{\prime}\right)$ association, the line curvature must be measured. Line profiles of telluric lines were recorded at various positions $j$ along the slit on flat-field observations, then relative shifts $\Delta i(j)=i^{\prime}(\lambda)-i(\lambda)$ in the dispersion direction of these profiles were derived for these positions $j$. Finally a cubic spline interpolation was used giving $i(j)$ at all positions for a given $\lambda$.

The characteristics of the polarized radiation are found by measuring its Stokes parameters. The orientation of the $X Y Z_{S}$ and $X^{\prime} Y^{\prime} Z_{S}$ depends on the instrumental setting. The $O X^{\prime}$ axis defining with $O Y^{\prime}$ the Stokes parameter $U$ was either parallel (July 18th 2000) or perpendicular (June 15th 2001) to the spectrograph entrance slit direction. The direction of the linear polarization was found using the classical formula $\Phi=$ $0.5 \arctan (U / Q)$ for $Q>0$ or $\Phi=0.5 \arctan (U / Q) \pm 90^{\circ}$ for $Q<0$, where $\Phi$ is the angle (defined to $\pm 180^{\circ}$ ) between the $O X$ axis defining, with $O Y, Q$ and the linear polarization direction (Notice that in reality in THEMIS the Stokes $U$ parameter is defined using a $X^{\prime} Y^{\prime} Z_{S}$ coordinate system derived from $X Y Z_{S}$ by a rotation of $-90^{\circ}$ and not $+90^{\circ}$ as usual. So, in

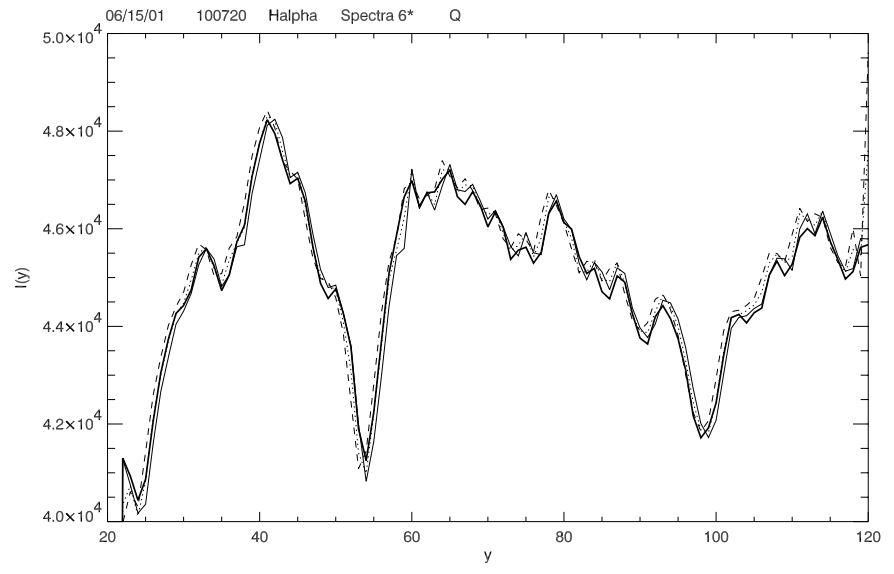

Fig. A.1. Intensity $I_{\Delta \lambda}$ in the far wings of the $\mathrm{H} \alpha$ line integrated over $20 \mathrm{~nm}$ as a function of distance $y$ (in pixels) along the spectrograph entrance slit, at 10:07:34 UT. The $y$ scale of the $I+S$ image has been translated by $-(142+\mathrm{d} y)$ pixels, where $\mathrm{d} y$ is a fraction of pixels. $I(y)$ in full and thick line corresponds to the image $I-S$. The other plots represent $I_{\Delta \lambda}(y-(142+\mathrm{d} y))$ given by the $I+S$ image. Three values of $\mathrm{d} y$ have been used, 0.(thin full line), +0.2 (thin dotted line)and +0.4 (thin dashed line).

order to reduce THEMIS data the sign of $U$ must be changed in all the equations given in this paper).

The Stokes parameters $U$ and $Q$ are measured by rotating an ensemble of two quarter wave-plates that act as a half-wave plate. We assume below that the positioning of the two quarter wave-plates is perfect and consider their ensemble as a single wave-plate (half-wave if the two quarter wave-plates are perfect). The intensities $I_{X}$ and $I_{Y}$ transmitted by two linear polarizers transmitting the intensity components along the $O X$ and $O Y$ directions respectively and located after the retarder system are:

$$
\begin{aligned}
I_{X}= & \frac{1}{2}\left(I-\frac{Q}{4}((1+\cos \delta)+(1-\cos \delta) \cos 4 \theta)\right. \\
& \left.+\frac{U}{2}(1-\cos \delta) \sin 4 \theta-\frac{V}{2} \sin 2 \theta \sin \delta\right) \\
I_{Y}= & \frac{1}{2}\left(I+\frac{Q}{4}((1+\cos \delta)+(1-\cos \delta) \cos 4 \theta)\right. \\
& \left.-\frac{U}{2}(1-\cos \delta) \sin 4 \theta+\frac{V}{2} \sin 2 \theta \sin \delta\right)
\end{aligned}
$$

where $\delta$ is the retardation of the rotating wave-plate and $\theta$ is the angle between the $O X$ axis and the slow axis of this wave-plate. The Stokes parameters are derived from the $I_{Y}-I_{X}$ difference:

$$
\begin{aligned}
I_{X}-I_{Y}= & -\frac{Q}{2}((1+\cos \delta)+(1-\cos \delta) \cos 4 \theta) \\
& \left.+\frac{U}{2}(1-\cos \delta) \sin 4 \theta-\frac{V}{2} \sin 2 \theta \sin \delta\right) .
\end{aligned}
$$

For a perfect half-wave plate, the $I_{Y}-I_{X}$ signal varies as:

$I_{X}-I_{Y}=-Q \cos 4 \theta+U \sin 4 \theta$

since the $(1+\cos \delta)$ term in Eqs. (A.2) is null. When the effective wave-plate is not exactly half-wave, an extra $\frac{Q}{2}(1+\cos \delta)$ 
term independent of $\theta$ is present in all measurements of $U$ and $Q$. Fortunately its relative importance, even for a retardation differing by $30^{\circ}$ from $180^{\circ}$ (i.e. $15^{\circ}$ on each quarter waveplate), does not exceed $7.2 \%$ of $U$ or $Q$ (assuming the same order of magnitude for both which was the case in our measurements), i.e. no more than $0.5 \%$ of the intensity. This error may affect slightly the determination of the polarization direction. It does not generate a false $Q$ polarization signal, just its value may be slightly affected.

For positions of the wave-plate such that $\sin 2 \theta$ differs from zero, which was the case for the $U$ measurements, $V$ cross-talk can be present. The $V$ cross-talk coefficient $(\sin 2 \theta \sin \delta) /((1-$ $\cos \delta$ )) reaches $19 \%$ for $\delta$ equal to $150^{\circ}$ and $12 \%$ for $\delta$ equal to $150^{\circ}$. The positions of the the wave-plate used to deriv Stokes $Q$ were such that $\sin 2 \theta$ was equal to zero and no $V$ cross-talk affected the $Q$ measurements.

The most serious source of spurious polarization signal is the intensity cross-talk present when the associated pixels $(i, j)$ and $\left(i^{\prime}, j^{\prime}\right)$ in the two $I+S$ and $I-S$ images do not correspond to the same location on the sun. So, as explained above, we made a relative positioning in the $y$ direction of these images with an accuracy of 0.08 arc second as shown in Fig. A.1. We point out that no relative positioning could be found that would lead to a null $(I+S)-(I-S)$ signal in the line profile.

\section{References}

Fárník, F., Garcia, H., \& Karlický, M. 2001, Sol. Phys., 201, 357

Feautrier N. 2002, private communication

Hénoux, J.-C., \& Vogt E. 1998, Plasma Scr., 78, 60

Heyvaerts, J. 1981, in Solar Flare Magnetohydrodynamics, ed. E. R. Priest (New York: Gordon and Breach Science Publ.)

Hoyng, P., Brown, J. C., \& Van Beek, H. F. 1976, Sol. Phys., 48, 197 Karlický, M. 1993, Sol. Phys., 145, 137

Karlický, M., \& Hénoux, J.-C. 2002, A\&A, 383, 713

Machado, M. E., Avrett, E. H., Vernazza, J. E., \& Noyes, R. W. 1980, A\&A, 242, 336

Norman, C., \& Smith, R. 1978, A\&A, 68, 145

Percival, I. C., \& Seaton, M. J. 1959, Phil. Trans. R. S. London A, 251, 113

Rowland, L., \& Vlahos, L. 1983, A\&A, 142, 219

Semel, M., 1980, A\&A, 91, 369

van den Oord, G. H. 1990, A\&A, 234, 496 